\title{
Differential gain in InP-based strained layer multiple quantum well lasers
}

\author{
D. Nichols and P. Bhattacharya \\ Solid State Electronics Laboratory, Department of Electrical Engineering and Computer Science, \\ University of Michigan, Ann Arbor, Michigan 48109-2122
}

(Received 18 March 1992; accepted for publication 24 August 1992)

\begin{abstract}
Compressive biaxial strain has been predicted to enhance the small-signal modulation bandwidth of quantum well lasers, owing to increased differential gain in these devices. However, the effect of tensile strain on these devices is less clear. We have investigated the effects of both compressive and tensile strain on the differential gain for multiple quantum well lasers with $\operatorname{In}_{x} \mathrm{Ga}_{1-x}$ As quantum wells for $0.33 \leqslant x \leqslant 0.73$. We observe markedly increased differential gain for both compressive and tensile strain, indicating that large modulation bandwidths can be obtained in both cases.
\end{abstract}

Biaxial strain has been shown to produce significantly enhanced performance characteristics in InP-based multiple quantum well lasers. ${ }^{1}$ These improvements are a result of changes in the band structure induced by strain which lead to decreased hole mass and decreased density of states at the band edge. ${ }^{2}$ This decrease in the band edge density of states leads also to a predicted increase in the differential gain for strained devices versus their lattice-matched counterparts. Up to the present time, however, there have been few experimental results on the differential gain in strained multiple quantum well lasers. In particular, the effects of biaxial tensile strain on differential gain is not known at all. In this letter, we present experimental data on the effect of strain on the differential gain in $\operatorname{InP} / \operatorname{In}_{x} \mathrm{Ga}_{1-x} \mathrm{As}(50 \AA)$ / InGaAsP $(0.33<x<0.73)$ multiple quantum well lasers. It is important to note that in the present study, the well thicknesses have not been altered to give constant wavelength, but are the same for all In compositions. This was done in order to isolate the effect of strain on the differential gain. The results show significantly enhanced differential gain for compressively strained and tensilely strained devices over lattice-matched devices, indicating greater potential small-signal modulation bandwidths.

The modal gain spectrum of a Fabry-Perot semiconductor laser may be derived from the measurement of its spontaneous emission spectrum. ${ }^{3,4}$ The optical attenuation coefficient, $\alpha$ may be related to the constructive and destructive intensities using the relation

$$
\Gamma \alpha(\lambda)=\frac{1}{L}\left(\ln \frac{r^{1 / 2}(\lambda)+1}{r^{1 / 2}(\lambda)-1}+\ln R\right),
$$

where $L$ is the cavity length, $r(\lambda)$ is the ratio of the maximum constructive intensity to the minimum destructive intensity at wavelength $\lambda, \Gamma$ is the optical confinement factor, and $R$ is the mirror reflectivity. In this equation, $\alpha$ refers to the net loss, i.e., the guide loss minus the optical gain. Furthermore, we assume that a linear variation of the gain with the injected carriers is valid over a reasonably broad range of carrier densities. ${ }^{5}$ These measurements were carried out on devices with cavity lengths ranging from 225 to $400 \mu \mathrm{m}$. However, Tatham et al. ${ }^{6}$ recently carried out measurements of the differential gain on lattice-matched devices with cavity lengths varying from $70 \mu \mathrm{m}$ all the way to $1 \mathrm{~mm}$ and similar numbers of wells. The reported effect of the cavity length was significantly smaller than the effect of strain measured here.

Spectral outputs below threshold were measured for these devices at various levels of drive current. From these spectra, the ratio of the peak envelope function to the valley envelope function may be calculated as a function of $\lambda$ and the results used to calculate the gain spectra of the device for the various levels of drive current. The modal gain spectra for several levels of drive current are shown in Fig. 1(a). From these data, one may plot the variation of the peak modal gain with current and then, from the slope of this characteristic, the differential gain. In making this conversion, the optical confinement factor, which was calculated numerically using a weighted index method, was assumed constant for all the structures. Similarly, the spontaneous carrier lifetime should be independent of strain and therefore we use a value of 2 ns for each composition.

The peak modal gain as a function of carricr density is shown in Fig. 1(b) for both lattice matched devices and devices with strained quantum wells. The peak modal gain for the devices with $33 \%$ In in the wells (open circles) is in the TM polarization whereas the other compositions are TE polarized. This demonstrates a significant shift of the light hole subband with respect to the heavy hole subband. In a lattice-matched quantum well, the light hole band is above the heavy hole band in energy because of the inverse mass dependence of the energy shift. The application of compressive strain increases this separation. However, tensile strain moves the band closer together until they cross at an In composition of approximately 0.46 . Subsequently, i.e., for devices with $33 \%$ In in the wells, the light hole band is the lower in energy than the heavy hold band. This is further evidenced by the output polarization characteristics of these lasers as a function of strain shown in Fig. 2. We see that for compositions of $48 \%$ or higher the emission is very nearly completely TE polarized, indicating lasing in the heavy hole band. However, for devices with $43 \%$ and $33 \%$ In in the wells, the light is roughly $33 \% \mathrm{TE}$ and $67 \% \mathrm{TM}$, indicative of the fact that a light hole is twice as 


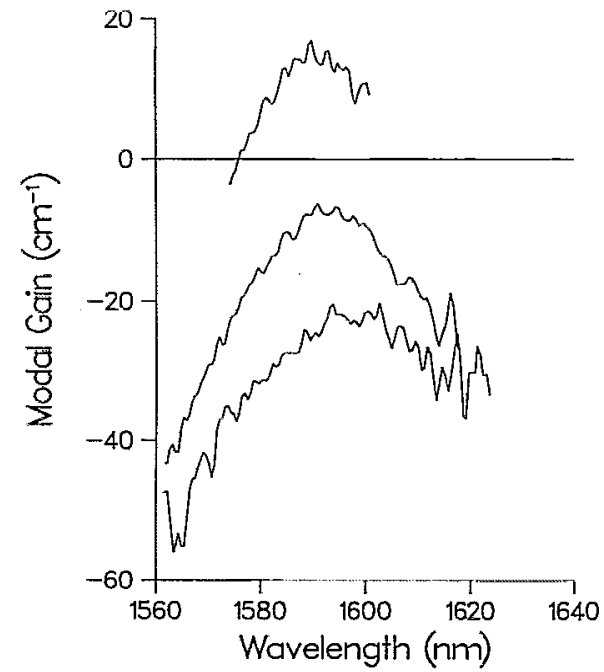

(a)

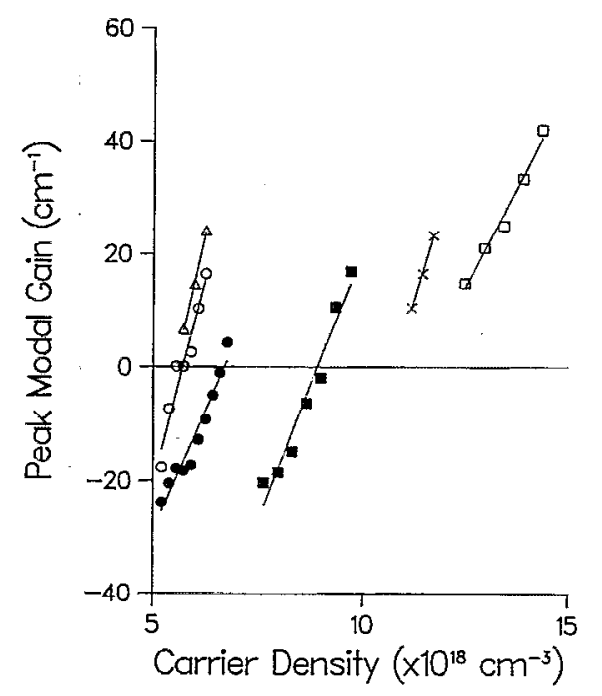

(b)

FIG. 1. (a) Calculated modal gain spectra for devices with $63 \%$ In in the wells for drive currents of 220,250 , and $280 \mathrm{~mA}$. (b) Peak modal gain for strained and lattice-matched devices. The $X$ 's are peak TM gain for $x=0.33$, all other symbols are peak TE gain. Open squares are for $x$ $=0.53$, shaded circles are for $x=0.58$, shaded squares are for $x=0.63$, open circles are for $x=0.68$, and open triangles are for $x=0.73$.

likely to couple to a TM polarized photon as to a TE photon.

The differential gain as a function of In composition is shown in Fig. 3. We see that the differential gain changes from a minimum of around $4 \times 10^{-16} \mathrm{~cm}^{2}$ at lattice match to roughly double that for both $20 \%$ excess and deficient In. This indicates that one can expect to see markedly improved small signal modulation bandwidths in strained devices versus lattice-matched devices.

We have fabricated ridge devices on semi-insulating substrates with the same structures and have tested their small signal response for lattice matched devices and devices with $63 \%$ In in the wells and found that the modulation bandwidths increased from 3 to $5 \mathrm{GHz}$. Several other workers ${ }^{7,8}$ have also found improved modulation

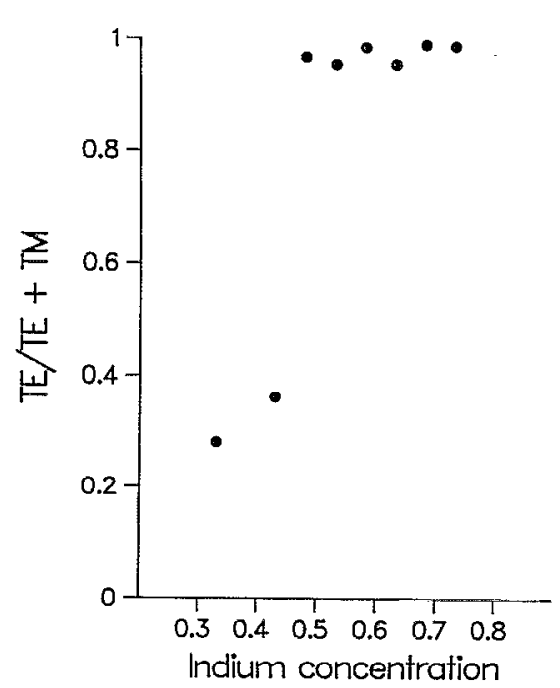

FIG. 2. Measured output polarization as a function of In composition in the wells.

properties in strained-layer lasers in both the $\operatorname{InP}$ and GaAs-based systems as well. One can gain insight into the available small-signal modulation bandwidth of a semiconductor laser by examining its relaxation oscillation frequency. This frequency is given by ${ }^{9}$

$$
f_{r}=\frac{1}{2 \pi} \sqrt{\frac{c(\delta g / \delta n) S}{n \tau_{p}}},
$$

where $S$ is the intracavity photon density, $n$ is the refractive index, $\tau_{p}$ is the photon lifetime and $\delta g / \delta n$ is the differential gain. Assuming an output power of $2 \mathrm{~mW}$ at $1.5 \mu \mathrm{m}$, we calculate a resonance frequency of $2.9 \mathrm{GHz}$ for latticematched devices, $3.4 \mathrm{GHz}$ for $x=0.63,4.4 \mathrm{GHz}$ for $x$ $=0.73$, and $4.1 \mathrm{GHz}$ for $x=0.33$ with the measured values of the differential gain. These results correspond well to the observations we have made in our laboratory, but are by no

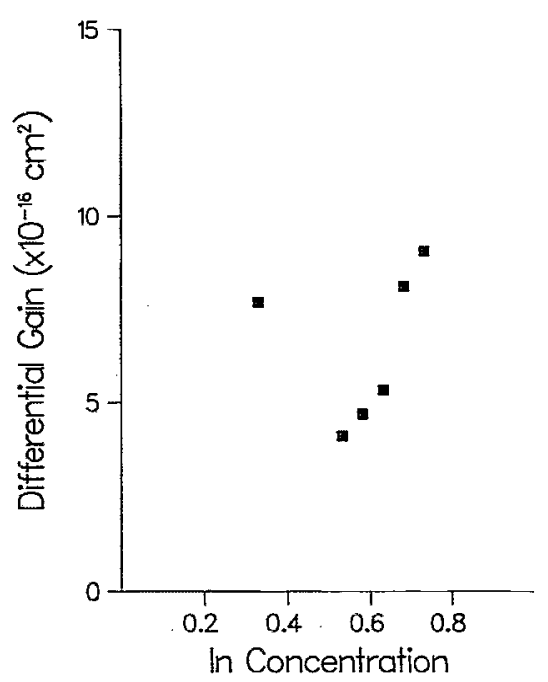

FIG. 3. Differential gain as a function of In composition in the wells. 
means indicative of an upper limit of the modulation bandwidth as this scales as the square root of the output power.

This work is being supported by the Office of Naval Research under Grant N00014-90-J-1831 and the Army Research Office (URI Program) under Contract DAAL03-86-K007. The authors wish to acknowledge Gordon Munns and Marc Sherwin for assistance with the growth of the layers and Glen Feak for useful discussions and help with the measurements.

${ }^{1}$ D. Nichols, M. Sherwin, G. Munns, J. Pamulapati, J. Leohr, J. Singh, and P. Bhattacharya, IEEE J. Quantum Electron. 28, 1239 (1992).
${ }^{2}$ J. P. Loehr and J. Singh, IEEE J. Quantum Electron. 27, 708 (1991). ${ }^{3}$ B. W. Hakki and T. L. Paoli, J. Appl. Phys. 44, 4113 (1973).

${ }^{4}$ B. W. Hakki and T. L. Paoli, J. Appl. Phys. 46, 1299 (1975).

${ }^{5}$ G. P. Agrawal and N. K. Dutta, Long-Wavelength Semiconductor Lasers (Van Nostrand-Reinhold, New York, 1986), p. 92.

${ }^{6}$ M. C. Tatham, I. F. Lealman, C. P. Seltzer, L. D. Westbrook, and D. M. Cooper, IEEE J. Quantum Electron. 28, 408 (1992).

${ }^{7}$ L. F. Lester, W. J. Schaff, S. D. Offsey, and L. F. Eastman, IEEE Photon. Tech. Lett. 3, 403 (1991).

${ }^{8}$ T. Fukushima, J. E. Bowers, R. A. Logan, T. Tanbun-Ek, and H. Temkin, Appl. Phys. Lett. 58, 1244 (1991).

${ }^{9}$ I. Suemune, L. A. Coldren, M. Yamanishi, and Y. Kan, Appl. Phys. Lett. 53, 1378 (1988). 